\title{
NUMERICAL INVESTIGATION OF DESTABILIZATION OF SUPERCRITICAL ROUND TURBULENT JETS USING LARGE EDDY SIMULATION
}

\author{
T. Schmitt ${ }^{1}$, A. Ruiz ${ }^{1}$ L. Selle ${ }^{2}$, and B. Cuenot $^{1}$ \\ ${ }^{1} \mathrm{CERFACS}$ \\ Av. G. Coriolis 42, Toulouse cedex 31057, France \\ ${ }^{2}$ Université de Toulouse \\ INPT, UPS \\ Institut de Mécanique des Fluides de Toulouse (IMFT) \\ Allée Camille Soula, Toulouse 31400, France
}

\begin{abstract}
In rocket engines, dense oxygen is injected in a high-pressure environment, above its critical pressure. Oxygen temperature varies from a subcritical value at injection to a supercritical (SC) value in the burnt gases. Both the Vulcain 2 engine and Space Shuttle main engine use this injection mode. Thermodynamics, mixing, and transport properties are no longer those of a perfect-gas mixture. The present study uses the Large Eddy Simulation (LES) code AVBP, developed at CERFACS to simulate such jets. Dense fluid flows are modeled by the use of a cubic equation of state, in conjunction with appropriate viscosity and thermal conductivity coefficients. A nitrogen round jet at SC pressure injected in a gaseous reservoir is simulated. Two cases are considered, one experiencing a transcritical (TC) injection (high-density injection), while the other one is injected at SC temperature (low-density injection). Mixing efficiency is studied and the stabilizing effect of the density gradients is identified. Results are in good agreement with available measurements. The funding for this research is provided by Snecma and CNES (Centre National d'Etudes Spatiales).
\end{abstract}

\section{INTRODUCTION}

In rocket engines, dense oxygen is injected in a high-pressure environment, above its critical pressure. Oxygen temperature varies from a subcritical value at injection to an $\mathrm{SC}$ value in burnt gases. Both the Vulcain 2 engine and Space

This is an Open Access article distributed under the terms of the Creative Commons Attribution-Noncommercial License 3.0, which permits unrestricted use, distribution, and reproduction in any noncommercial medium, provided the original work is properly cited. 
Shuttle main engine use this injection mode. In such conditions, fluid behavior may strongly differ from that of a perfect gas $[1,2]$. In order to gain insight into the physics of high-pressure flows in complex configurations, the development of a computational fluid dynamics (CFD) code for predicting the flow features is of great interest and of great need, especially for long lasting development cycles as in the aerospace industry. For this reason, Snecma which is the prime contractor for the European launcher Ariane 5 cryogenic propulsion systems and CNES which is the government agency responsible for shaping and implementing the space policy of France in Europe, have launched a program on the development of LES for SC combustion.

High-pressure cryogenic round jets were experimentally studied by AFRL (Air Force Research Laboratory) and DLR (Deutsches Zentrum fur Luft- und Raumfahrt) [3-12]. Both laboratories noticed a strong impact of pressure on the jet topology. Indeed, as pressure reaches its critical value, the classical behavior of two-phase jets is no longer observed, and dense fluid is continuously dissolved in ambient gas, leading to the apparition of high density gradients between the dense cold jet and the ambient gas, with no evidence of droplet formation. Part of the DLR experimental results on cryogenic round jets has served as a test case, referenced as RCM-1, in the 2nd International Workshop on Rocket Combustion Modeling [13].

Supercritical fluid behavior was also studied numerically, in particular with Direct Numerical Simulation (DNS) of mixing layers, showing the role of density gradients on the global layer stability and turbulence characteristics [14]. Only few LES studies have been performed under SC-pressure conditions. An extensive review of the nonreacting LES studies up to 2006 can be found in [15]. A single nitrogen round jet was studied by Zong et al. [16, 17]. The stabilizing effect of the density gradient, and its role on turbulent energy redistribution along the mixing layer was identified. Zong and Yang [18] also simulated a coaxial injection of oxygen and methane while shear coaxial $\mathrm{LOx} / \mathrm{GH}_{2}$ jet flame at $\mathrm{SC}$ pressure was investigated by both Oefelein [19] and Matsuyama et al. [20]. To the authors' knowledge, the studies by Zong et al. [16, 17], who simulated highpressure round jets from AFRL experiments, are the only unstationary analyses of a three-dimensional (3D) nonreactive cryogenic round jet at high Reynolds number. RCM-1 test case has been simulated using Reynolds-averaged NavierStokes (RANS) approach by Branam and Mayer [9] and Cheng and Farmer [21]. The aim of the current study was to describe the destabilization processes for a nitrogen jet under SC conditions.

For the proper description of SC fluid dynamics, two major modifications must be made to the standard low-pressure Navier-Stokes equations:

- an Equation Of State (EOS) that accounts for real-gas effects must be implemented; and

- transport models for mass and heat transfers must be modified. 
The EOS can be considered as the cornerstone of SC fluid modeling since it ensures the accuracy of the method in a quiescent fluid. Indeed, basic thermodynamic variables such as the density or the pressure dependence of the specific heats are directly driven by the EOS. From a practical point of view, the EOS must compromise between accuracy and computational cost, leading to cubic EOS.

This paper is organized as follows: real-gas models are presented in section 2 , then the flow configuration and the numerical setup of the present simulation are presented in section 3. Finally, the results are discussed in section 4 .

\section{REAL-GAS MODEL}

In this section, two key ingredients of the numerical simulation of SC flows are presented: the EOS and the model for transport phenomena. The PengRobinson (PR) EOS [22] was chosen for this work and has been implemented in the AVBP LES solver [23]. For a single species, it reads:

$$
P=\frac{\rho r T}{1-b \rho}-\frac{\rho^{2} a(T)}{1+2 b \rho-b^{2} \rho^{2}}
$$

where $P$ is the pressure; $T$ is the temperature; $\rho$ is the density; and $r=R / W$ with $R$ being the universal gas constant and $W$ the molar mass. The coefficients $a(T)$ and $b$ are defined as:

$$
a(T)=0.457236 \frac{\left(r T_{c}\right)^{2}}{P_{c}}\left[1+c\left(1-\sqrt{\frac{T}{T_{c}}}\right)\right]^{2} ; \quad b=0.077796 \frac{r T_{c}}{P_{c}}
$$

where $P_{c}$ is the critical pressure; $T_{c}$ is the critical temperature; and additional parameter $c$ is defined as a function of the acentric factor $\omega_{\mathrm{ac}}[24]$ by

$$
c=0.37464+1.54226 \omega_{\mathrm{ac}}-0.26992 \omega_{\mathrm{ac}}^{2}
$$

with $\omega_{\mathrm{ac}}=0.0372$ for $\mathrm{N}_{2}$. Equation (1) is then used for a consistent derivation of the pressure dependence of thermodynamic coefficients (specific heat, speed of sound, etc.), similarly to the procedure used by Miller et al. [25], whereas the low-pressure reference values are provided by the JANAF thermochemical tables [26]. The performance of this model for the present study is illustrated in Fig. 1 by comparing the density and the constant-pressure specific heat $C_{p}$ with the data from the NIST database [27] at 40 bar and within the temperature range of the experimental conditions presented in section 3 .

The relative error is less than $3 \%$ for the density and $10 \%$ for the specific heat, except near the pseudoboiling line (see Fig. $1 b$ around $130 \mathrm{~K}$ ) where the 


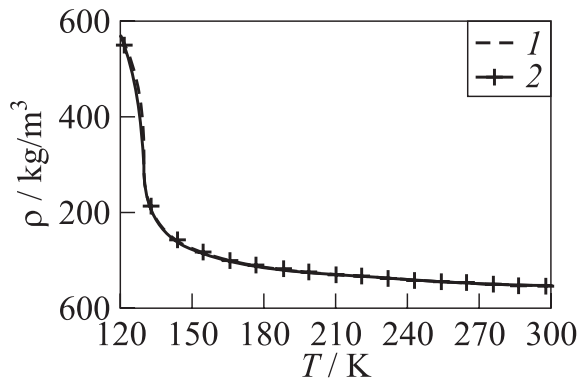

(a)

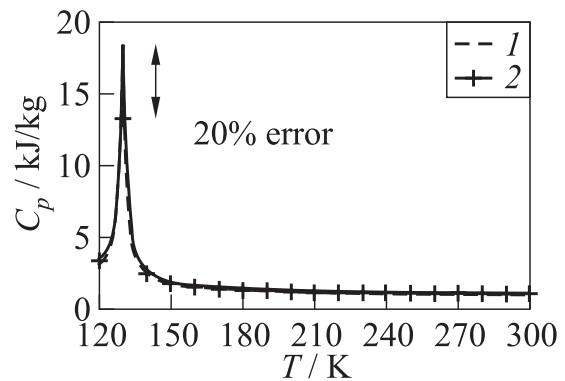

(b)

Figure 1 Validation of the EOS and specific heat for $\mathrm{N}_{2}$ at $40 \mathrm{bar}\left(P_{\mathrm{N}_{2}}=34 \mathrm{bar}\right.$, $\left.T_{c_{\mathrm{N}_{2}}}=126 \mathrm{~K}\right): 1$ - NIST database; and 2 - real-gas model, based on the PR EOS [22]

error increases locally to $5 \%$ for the density and $20 \%$ for the specific heat. The pseudoboiling temperature $T_{\mathrm{pb}}$ is the temperature for which, at a given pressure, the constant-pressure specific heat $C_{p}$ reaches its maximum; it is the prolongation of the gas/liquid phase-change line [12].

Bellan and Harstad [28] proposed a formulation of transport phenomena under SC conditions consistent with the kinetic theory at low pressure. For the configuration considered in this work (see section 3), many simplifications can be made. As a matter of fact, for single-species calculations, the only term that remains in the heat flux $q_{i}$ is the classical Fickian contribution:

$$
q_{i}=\lambda \frac{\partial T}{\partial x_{i}}
$$

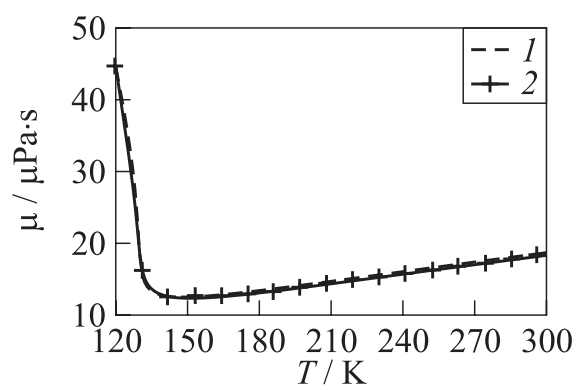

(a)

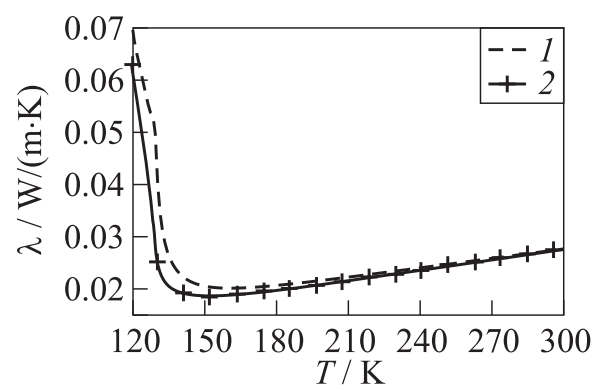

(b)

Figure 2 Validation of the transport coefficients: (a) viscosity; and $(b)$ thermal conductivity $(1$ - NIST database; and $2-[29])$ 
where $\lambda$ is the thermal conductivity. The method proposed by Chung et al. [29] is used to compute the transport coefficients: the thermal conductivity and the dynamic viscosity $\mu$. This method is based on the kinetic theory of gases, empirically corrected at high pressures. These coefficients are compared favorably with the NIST database within the thermodynamic conditions of this study (Fig. 2), with a mean relative error of $3 \%$ for the thermal conductivity and $0.6 \%$ for the dynamic viscosity, over the 120-300 K temperature range. Altogether, this model provides a quantitative evaluation of thermodynamic variables over a wide range of pressure (not shown here) and temperature. Noteworthy, it naturally degenerates toward perfect-gas behavior when the pressure is decreased.

\section{FLOW CONFIGURATION}

The configuration for the numerical simulation is the experimental setup $[9,10]$. It consists of a single round jet $(2.2 \mathrm{~mm}$ in diameter) injected in a cylindrical chamber (122 $\mathrm{mm}$ in diameter) pressurized at 39.7 bar at a temperature of $298 \mathrm{~K}$ (Fig. 3). As the chamber diameter is 60 times larger than the injector diameter, the jet is believed to behave as a free jet.

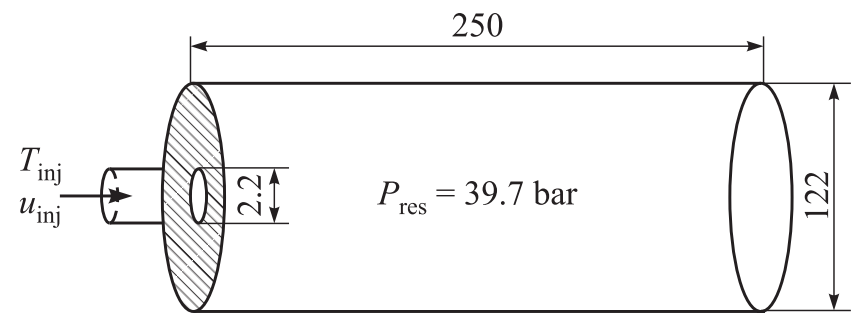

Figure 3 Sketch of the configuration. Dimensions are in millimeters

\subsection{Thermodynamic Conditions}

Two cases with different inlet temperatures were computed and are presented in Table 1 . The two cases are numbered according to $[9,10]$.

Table 1 Operating conditions for cases 3 and 4 of $[9,10]$

\begin{tabular}{cccccc}
\hline Case & $T_{\text {inj }}[\mathrm{K}]$ & $u_{\text {inj }}[\mathrm{m} / \mathrm{s}]$ & $T / T_{\mathrm{pb}}$ & $\rho_{\text {inj }} / \rho_{\infty}$ & $\mathrm{Re}_{\text {inj }}$ \\
\hline 3 & 126.9 & 4.9 & 0.98 & 9.6 & $1.7 \cdot 10^{5}$ \\
4 & 137 & 5.4 & 1.06 & 3.7 & $1.6 \cdot 10^{5}$ \\
\hline
\end{tabular}




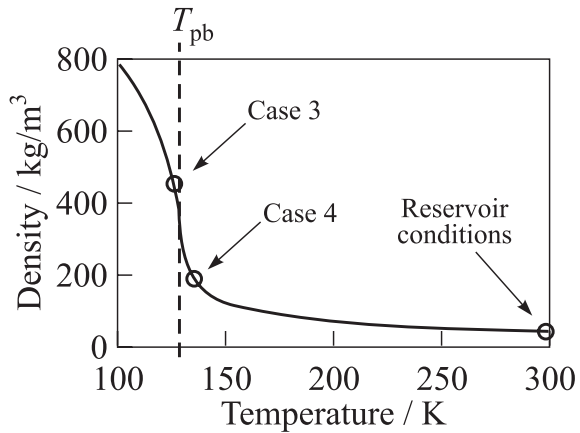

(a)

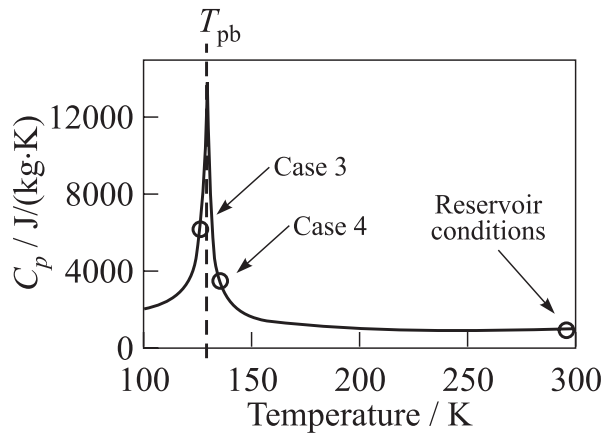

(b)

Figure 4 Injection conditions for cases 3 and 4: (a) density; and (b) constant-pressure specific heat

Case 3 is a so-called "TC" injection: the injected fluid is evolving from a liquid-like state (high density) to a gas-like state (low density) after being heated up by the ambient warm gas. A large amount of energy is needed to change the jet temperature, as indicated in the $C_{p}$ peak near the pseudoboiling temperature $T_{\mathrm{pb}}$ in Fig. $4 b$.

The injection temperature of case 4 is very similar to case 3 ; however, it is above the pseudoboiling temperature $\left(T_{\mathrm{pb}}=129.5 \mathrm{~K}\right.$ at $39.7 \mathrm{bar}$ for nitrogen $)$, which reduces the difference with the perfect-gas case. Hence, case 4 is merely qualified as "SC" injection in the following.

Because of the strong changes in dynamic viscosity near the pseudoboiling temperature (see Fig. 2a), the Reynolds numbers at injection for cases 3 and 4 are very close: $\operatorname{Re}_{\mathrm{inj}} \sim 160000$.

\subsection{Numerical Setup: Mesh and Boundary Conditions}

The computational domain used in the present 3D simulation corresponds to the experimental setup of Branam and Mayer [9]. A longitudinal cut of the mesh is displayed in Fig. $5 a$. The grid is finest near the injector, with a constant characteristic cell size of $0.1 \mathrm{~mm}$ over a distance of almost 10 diameters. This zone is followed by a smoothly coarsening region. The mesh, which is the same for both cases, contains 950000 points and 5500000 tetrahedra. The boundary conditions used in the present simulation are presented in Fig. $5 b$. The pressure in the reservoir is maintained by a nonreflecting outlet with a target pressure using the NSCBC (Navier-Stokes Characteristic Boundary Conditions) technique [30] made consistent with the real-gas EOS [31]. The walls near the injector are treated as adiabatic while the reservoir walls are kept at a constant temperature 


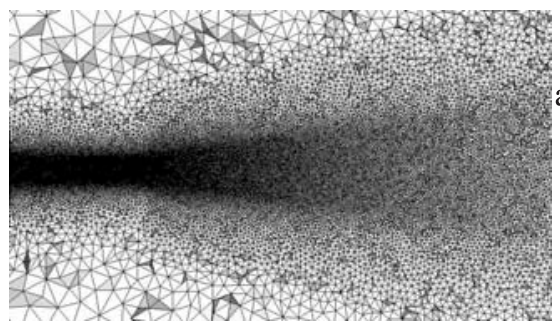

(a)
Imposed-velocity and temperature inlet

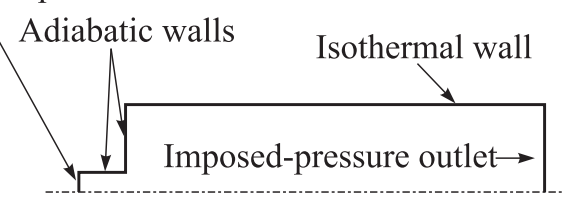

(b)

Figure 5 Numerical setup: (a) longitudiual cut of the computational domain mesh on a length of 30 injector diameters; and (b) boundary conditions

of $298 \mathrm{~K}$. The velocity and temperature are imposed at the inlet with turbulent perturbations prescribed by the procedure initially developed by Kraichnan [32, 33 and adapted to compressible flows [34] with an intensity of $2.5 \%$ of the mean flow.

The simulation is achieved using the TTGC (Two-step Taylor-Galerkin C) scheme [35]. This centered scheme is third order in time and space and has successfully been validated for both nonreacting and reacting flows [36]. Cryogenic jets at SC pressure are characterized by very steep density gradients which require specific numerical treatments. The steep density gradients are localized using a Jameson-like sensor [37] and artificial viscosity is added to the conservation equations in the regions indicated by this sensor. The highly nonlinear thermodynamics requires a consistent treatment of artificial fluxes to avoid spurious noise generation [38].

\section{RESULTS}

First, the quality of the present LES is assessed using the criterion proposed by Pope [39], which is presented in Fig. 6:

$$
M=\frac{k_{\mathrm{sgs}}}{k_{\mathrm{sgs}}+k_{\mathrm{res}}}
$$

where $k_{\text {sgs }}$ and $k_{\text {res }}$ are the subgrid scale turbulent energy and the resolved kinetic turbulent energy, respectively. The following expressions are used:

$$
\begin{aligned}
& k_{\mathrm{sgs}}=\left(\frac{\nu_{t}}{0.1 \delta}\right)^{2} ; \\
& k_{\mathrm{res}}=\frac{u_{\mathrm{rms}}^{2}+v_{\mathrm{rms}}^{2}+\omega_{\mathrm{rms}}^{2}}{2}
\end{aligned}
$$




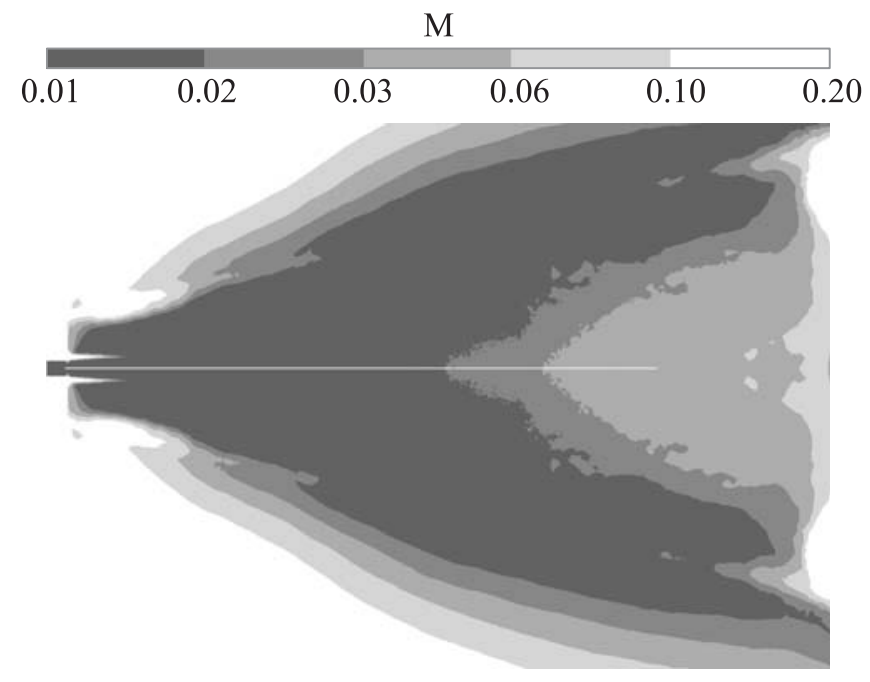

Figure 6 Estimation of the present LES quality M using the criterion of [39]

where $\nu_{t}, \delta, u_{\mathrm{rms}}, v_{\mathrm{rms}}$, and $w_{\mathrm{rms}}$ are the kinematic turbulent viscosity, the cell size, and the RMS values of the velocity components, respectively. The white line in Fig. 6 is 40 diameters long and indicates that more than $95 \%$ of the velocity fluctuations are resolved in this region. The coarsening of the mesh beyond the latter region makes this criterion reach $80 \%$. However, no results are analyzed there and the overall mesh quality is correct.

The centerline profile of density was experimentally measured in [9, 10] using two-dimensional (2D) Raman technique. These results are compared with the present numerical simulations in Fig. 7. For case 3, the computational results accurately predict the drop in centerline density despite a small (10\%) overestimation near the injector, which could be due to the systematic error of the Raman technique in very-high-density regions $[9,10]$. This overestimation does not come from a lack of precision of the PR EOS, since the density from the NIST database is $20 \mathrm{~kg} / \mathrm{m}^{3}$ higher than the density from the PR EOS, at the injection thermodynamic state. For case 4 , however, experimental and numerical results differ notably. This could come from a small discrepancy between the simulation and the experiment injection temperature, which implies a very large discrepancy between injection densities, since the inlet thermodynamic state is very close to the pseudoboiling point (see Fig. 4a). Finally, based on the centerline density profiles, one can evaluate the dense-core length $x_{\mathrm{DC}}$, which is defined, in the present study, as the downstream distance from the injector, where the density decreases below $99 \%$ of its injection value. It is found to be 7.9 diameters for case 3 and reduces to 5.1 diameters for case 4 . The mixing efficiency is thus 


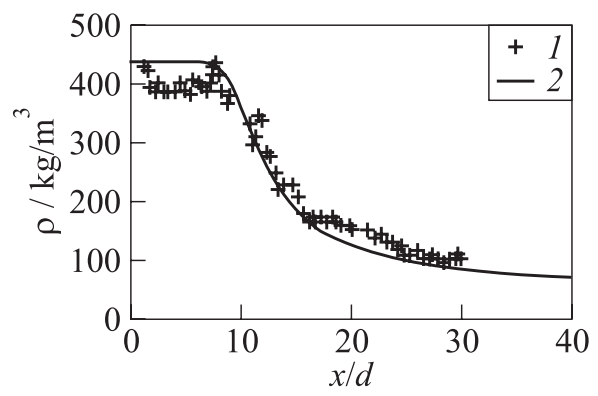

(a)

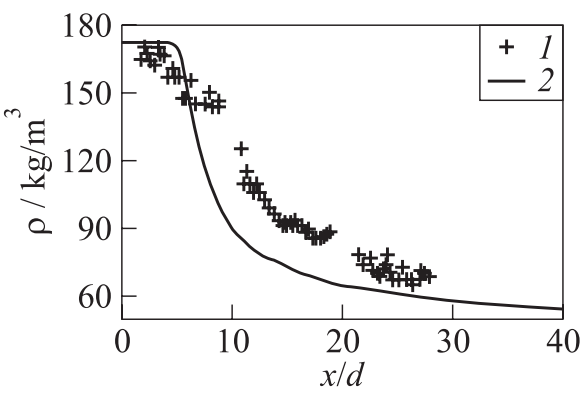

(b)

Figure 7 Comparison between experimental results $[9,10]$ (1) and present LES results $(2)$ for the centerline density in terms of normalized distance from the injector: (a) case 3 ; and $(b)$ case 4

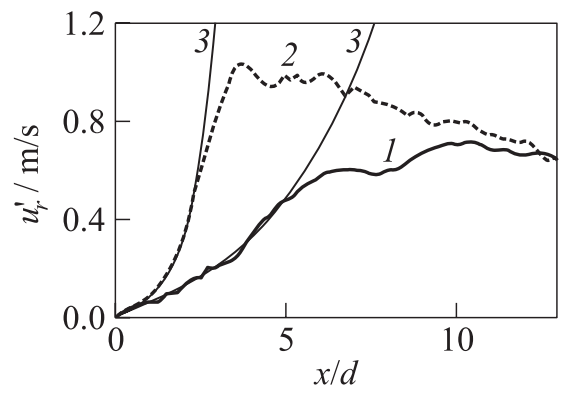

(a)

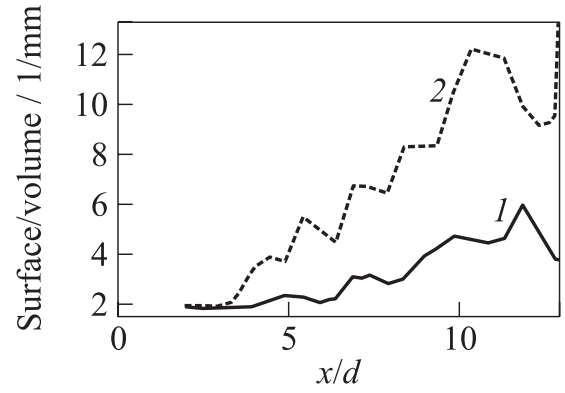

(b)

Figure 8 Mixing efficiencies: $(a)$ development of maximum radial velocity perturbations along shear layer; and $(b)$ instantaneous wrinkling of the dense core $(1$ - case 3 $\left(k_{i}=0.30\right) ; 2$ - case $4\left(k_{i}=1.36\right)$; and 3 - exponential fits $)$

reduced for a TC injection, which is consistent with phenomenological studies of high-pressure jets [12].

In order to assess mixing efficiency, the initial destabilization of the dense-core and its transition to fully developed turbulence is studied. Figure $8 a$ shows the axial development of maximum radial velocity perturbations $u_{r}^{\prime}$ along the shear layer. The development of $u_{r}^{\prime}$ in the near-injector region is much faster in case 4 than in case 3 . In case 4 , it reaches $20 \% u_{\text {inj }}$ at 2 diameters, while in case 3 , it reaches $10 \% u_{\text {inj }}$ at 13 diameters. An exponential fit of the initial growth of the velocity perturbation is made in order to obtain a spatial amplification coefficient $k_{i}\left(u_{r}^{\prime}=c s t+\exp \left(k_{i} x / d\right)\right)$. In case $4, k_{i}$ is approximately four times 


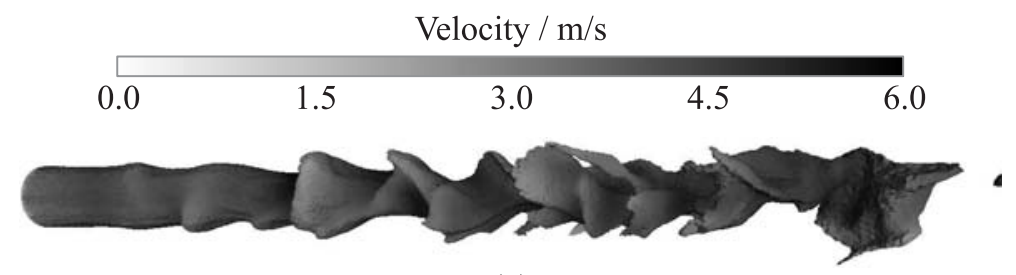

(a)

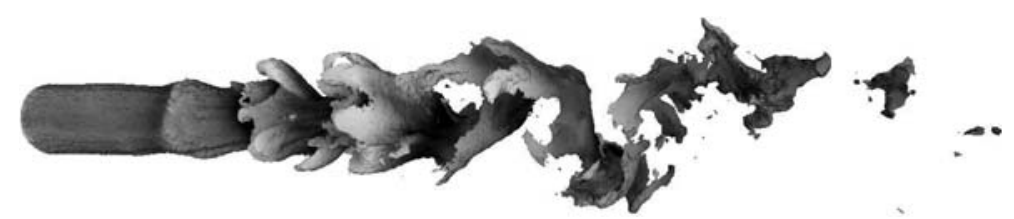

(b)

Figure 9 Isosurfaces at $\rho^{+}=0.5$ colored by velocity magnitude: $(a)$ case 3 ; and (b) case 4

as high as case 3 . The growth of the velocity perturbations appears similar to the Kelvin-Helmholtz instability and shows the stabilizing effect of the density gradient.

The effects of these velocity perturbations on the "dense fluid" are now investigated. The nondimensional density $\rho^{+}$is introduced:

$$
\rho^{+}=\frac{\rho-\rho_{\infty}}{\rho_{\text {inj }}-\rho_{\infty}}
$$

When $\rho^{+}=1$, the fluid has the injection density. When $\rho^{+}=0$, it has the reservoir gas density. An isosurface at $\rho^{+}=0.5$ colored by velocity magnitude (the darker the color, the higher velocity) is shown in Fig. 9, and the inner volume delimited by this isosurface is referred here to as the "dense fluid." In case 3 , the dense fluid is wrinkled by weak hydrodynamic instabilities and progressively disappears in the reservoir, whereas in case 4 , the dense fluid bursts at a few diameters downstream of the injector, similarly to gaseous jet disintegration. This is consistent with the radial velocity perturbation profiles shown in Fig. 8a. The wrinkling of the dense fluid is then assessed isolating one-diameter-long slices of the isosurfaces shown in Fig. 9 and computing the surface to volume ratio of these slices. The instantaneous longitudinal evolution of the dense-fluid wrinkling is plotted in Fig. $8 b$. The wrinkling of the transcritical dense fluid is much lower than the SC one, which reduces the exchange surface between warm and cold fluid, therefore reducing heat transfer. Together with the $C_{p}$ peak mentioned in subsection 3.1, this extends the TC dense-core length. 


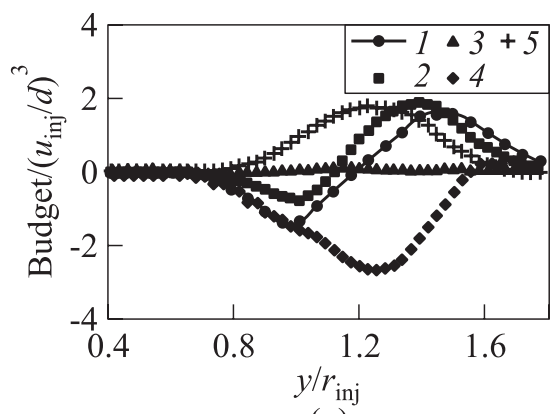

(a)

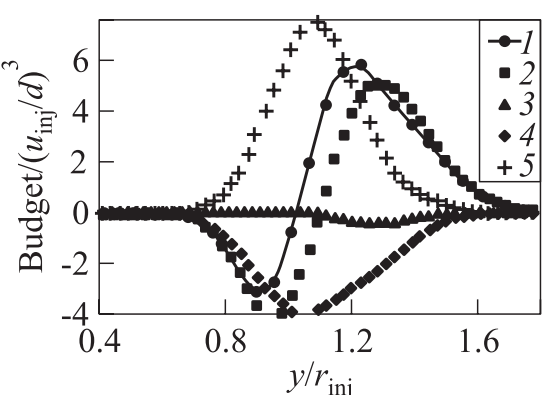

(b)

Figure 10 Enstrophy source terms in the transition zone of two jets: $(a)$ case 3, axial distance -4 diameters; and $(b)$ case 4 , axial distance -2.6 diameters $(1-$ total; 2 - baroclinic; 3 - dilatation; 4 - dissipation; and 5 - stretching)

In order to identify the phenomena responsible for the increase of velocity perturbations, the enstrophy $\omega^{2}$ balance equation is analyzed:

$$
\begin{aligned}
& \frac{D\left(\omega^{2}\right)}{D t}=2 \underline{\omega}(\underline{\omega \nabla}) \underline{u}-2(\underline{\nabla u}) \omega^{2}-2 \underline{\omega} \frac{\underline{\nabla} \rho \times \underline{\nabla} P}{\rho^{2}}+2 \underline{\omega \nabla} \times\left(\frac{1}{\rho} \underline{\nabla \tau}\right) \\
& \text { Stretching Dilatation Baroclinic Dissipation }
\end{aligned}
$$

where $\underline{\omega}=\underline{\nabla} \times \underline{U} ; \underline{U}$ is the velocity; and $\underline{\tau}$ is the viscous stress tensor. The average nondimensional source terms of the enstrophy equation are plotted in Fig. 10 for both cases. The radial profiles are taken at $x / x_{\mathrm{DC}}=0.5$ in order to get information about the dense-core destabilization. As vortex stretching is approximately compensated by viscous dissipation, the sum of all source terms in Eq. (2) closely follows the profile of the baroclinic torque in both cases, showing that they are both responsible for the damping and enhancement of the hydrodynamic instabilities in the dense and the light fluid, respectively. Similar observations are made by Zong [17] using LES and Okong'o and Bellan [40] using DNS. It appears that only the absolute value of the source terms are changed between case 3 and case 4 while their relative importance are approximately the same.

\section{CONCLUDING REMARKS}

Two simulations of SC round jets, representative of rocket-injection thermodynamic conditions, have been successfully undertaken with the LES code AVBP, using the PR EOS and appropriate transport coefficients. 
Mean centerline density profiles have been obtained and compared with the experimental results $[9,10]$. The results for the TC injection case (case 3, which has the most real-gas effects) show a very good agreement with experimental measurements, with the adequate decrease in the centerline density, and adequate dense-core length, whereas unexpected discrepancies exist for the SC injection case (case 4). The TC dense core is longer than the $\mathrm{SC}$ one, which is in qualitative agreement with other high-pressure experimental results [12].

The spatial amplification of the radial velocity perturbations along the shear layer have been computed and the initial growth rate of these perturbations is approximately a factor of four smaller in the TC case than in the SC case. The effects of these hydrodynamic perturbations on heat transfer have been identified through the evaluation of "dense fluid" wrinkling, which is less intense in case 3 than in case 4 . Hence, the extent of the TC dense core is not only due to the pseudophase change process but also to the reduced heat transfer induced by lower wrinkling.

Finally, the amplification mechanisms of hydrodynamic perturbations have been studied through the evaluation of the enstrophy-equation source terms. It has been shown that the driving phenomenon is the baroclinic torque which damps perturbations in the dense fluid while enhancing them in the light fluid.

\section{REFERENCES}

1. Hirschfelder, J., C. Curtis, and B. Bird. 1954. Molecular theory of gases and liquids. John Wiley \& Sons.

2. Poling, B. E., J. M. Prausnitz, and J.P. O'Connell. 2001. The properties of gases and liquids. McGraw-Hill.

3. Mayer, W., A. Schik, C. Schweitzer, and M. Schaffler. 1996. Injection and mixing processes in high pressure $\mathrm{LOx} / \mathrm{GH}_{2}$ rocket combustors. AIAA Paper No. 96-2620.

4. Mayer, W. and H. Tamura. 1996. Propellant injection in a liquid oxygen/gaseous hydrogen rocket engine. J. Propul. Power 12(6):1137-47.

5. Mayer, W., A. Schik, B. Vielle, C. Chaveau, I. Gökalp, and D. Talley. 1998. Atomization and breakup of cryogenic propellants under high pressure subcritical and supercritical conditions. J. Propul. Power 14(5):835-42.

6. Mayer, W., A. Ivancic, A. Schik, and U. Hornung. 1998. Propellant atomization in $\mathrm{LOx} / \mathrm{GH}_{2}$ rocket combustors. AIAA Paper No. 98Ц3685.

7. Oschwald, M. 1999. Supercritical nitrogen free jet investigated by spontaneous raman scattering. Exper. Fluids 27:497-506.

8. Chehroudi, B., D. Talley, and E. Coy. 2002. Visual characteristics and initial growth rate of round cryogenic jets at subcritical and supercritical pressures. Phys. Fluids 14(2):850-61.

9. Branam, R., and W. Mayer. 2003. Characterisation of cryogenic injection at supercritical pressure. J. Propul. Power 19(3):342-55. 
10. Mayer, W., J. Telaar, R. Branam, G. Schneider, and J. Hussong. 2003. Raman measurements of cryogenic injection at supercritical pressure. Heat Mass Transfer 39:709-19.

11. Mayer, W. O. H., and J. J. Smitht. 2004. Fundamentals of supercritical mixing and combustion of cryogenic propellants. In: Liquid rocket thrust chambers: Aspects of modeling, analysis, and design. Eds. V. Yang, M. Habiballah, J. Hulka, and M. Popp. Progress in astronautics and aeronautics ser. 200:339-68.

12. Oschwald, M., J. J. Smith, R. Branam, J. Hussong, A. Schik, B. Chehroudi, and D. Talley. 2006. Injection of fluids into supercritical environments. Combust. Sci. Technol. 178:49-100.

13. Telaar, J., G. Schneider, J. Hussong, and W. Mayer. 2001. Cryogenic jet injection: Description of test case RCM 1. 2nd Workshop (International) on Rocket Combustion Modeling Proceedings. Lampoldshausen, Germany.

14. Bellan., J. 2006. Theory, modeling and analysis of turbulent supercritical mixing. Combust. Sci. Technol. 178:253-81.

15. Zong, N., and V. Yang. 2006. Cryogenic fluid jets and mixing layers in transcritical and supercritical environments. Combust. Sci. Technol. 178:193-227.

16. Zong, N., H. Meng, S.-Y. Hsieh, and V. Yang. 2004. A numerical study of cryogenic fluid injection and mixing under supercritical conditions. Phys. Fluids 16:4248-61.

17. Zong., N. 2005. Modeling and simulation of cryogenic fluid injection and mixing dynamics under supercritical conditions. Ph.D. Thesis. Department of Mechanical and Nuclear Engineering, Pennsylvania State University.

18. Zong, N., and V. Yang. 2005. Numerical study of high-pressure oxygen/methane mixing and combustion of a shear coaxial injector. AIAA Paper No. 2005-152.

19. Oefelein, J.C. 2005. Thermophysical characteristics of shear-coaxial $\mathrm{LOx}-\mathrm{H}_{2}$ flames at supercritical pressure. Proc. Combust. Inst. 30(2):2929-37.

20. Matsuyama, S., J. Shinjo, Y. Mizobuchi, and S. Ogawa. 2006. A numerical investigation on shear coaxial $\mathrm{LOx} / \mathrm{GH}_{2}$ jet flame at supercritical pressure. 44th AIAA Aerospace Sciences Meeeting and Exhibit. Reno, Nevada.

21. Cheng, G.C., and R. Farmer. 2006. Real fluid modeling of multiphase flows in liquid rocket engine combustors. J. Propul. Power 22(6):1373.

22. Peng, D. Y., and D. B. Robinson. 1976. A new two-constant equation of state. Ind. Eng. Chem. Fund. 15(1):59-64.

23. Schonfeld, T., and M. Rudgyard. 1999. Steady and unsteady flows simulations using the hybrid flow solver AVBP. AIAA J. 37(11):1378-85.

24. Pitzer, K. S., D.Z. Lippmann, R. F. Curl, Jr., C. M. Huggins, and D. E. Petersen. 1955. The volumetric and thermodynamic properties of fluids. II. Compressibility factor, vapor pressure and entropy of vaporization. J. Amer. Chem. Soc. 77(13):3433-40.

25. Miller, R. S., K. G. Harstad, and J. Bellan. 2001. Direct numerical simulations of supercritical fluid mixing layers applied to heptane-nitrogen. J. Fluid Mech. 436:139.

26. NIST-JANAF Thermochemical Tables. 1998. National Institute of Standards and Technology. 
27. Lemmon, E. W., M. O. McLinden, and D. G. Friend. 2009. Thermophysical properties of fluid systems. NIST Chemistry Webbook. NIST standard reference database. 69.

28. Bellan, J., and K. Harstad. 2000. An all-pressure fluid drop model applied to a binary mixture: Heptane in nitrogen. Int. J. Multiphase Flow 26(10):1675-706.

29. Chung, T. H., M. Ajlan, L. L. Lee, and K. E. Starling. 1988. Generalized multiparameter correlation for nonpolar and polar fluid transport properties. Ind. Eng. Chem. Res. 27(4):671-79.

30. Poinsot, T., and S. Lele. 1992. Boundary conditions for direct simulations of compressible viscous flows. J. Comput. Phys. 101(1):104-29.

31. Okong'o, N., and J. Bellan. 2002. Consistent boundary conditions for multicompoment real gas mixtures based on characteristic waves. J. Comput. Phys. 176:330-44.

32. Kraichnan, R. H. 1970. Diffusion by a random velocity field. Phys. Fluids 13:22-31.

33. Smirnov, A., S. Shi, and I. Celik. 2001. Random flow generation technique for large eddy simulations and particle-dynamics modeling. Trans. ASME. J. Fluids Eng. 123:359-71.

34. Guezennec, N., and T. Poinsot. 2009. Acoustically nonreflecting and reflecting boundary conditions for vorticity injection in compressible solvers. AIAA J. 47:1709-22.

35. Colin, O., and M. Rudgyard. 2000. Development of high-order Taylor-Galerkin schemes for unsteady calculations. J. Comput. Phys. 162(2):338-71.

36. Selle, L., G. Lartigue, T. Poinsot, R. Koch, K.-U. Schildmacher, W. Krebs, B. Prade, P. Kaufmann, and D. Veynante. 2004. Compressible large-eddy simulation of turbulent combustion in complex geometry on unstructured meshes. Combust. Flame 137(4):489-505.

37. Jameson, A., W. Schmidt, and E. Turkel. 1981. Numerical solution of the Euler equations by finite volume methods using Runge-Kutta time stepping schemes. AIAA Paper No. 81-1259.

38. Schmitt, T., L. Selle, A. Ruiz, and B. Cuenot. 2010. Large-eddy simulation of supercritical-pressure round jets. AIAA J. 48(9):2133-44.

39. Pope., S. B. 2004. Ten questions concerning the large-eddy simulation of turbulent flows. New J. Phys. 6:35.

40. Okong'o, N. A., and J. Bellan. 2002. Direct numerical simulation of a transitional supercritical binary mixing layer: Heptane and nitrogen. J. Fluid Mech. 464:1-34. 